\title{
Numeration Systems: a Link between Number Theory and Formal Language Theory
}

\author{
Michel Rigo \\ Université de Liège, Institut de Mathématiques, Grande Traverse 12 (B 37), \\ B-4000 Liège, Belgium, M.Rigo@ulg.ac.be *
}

\begin{abstract}
We survey facts mostly emerging from the seminal results of Alan Cobham obtained in the late sixties and early seventies. We do not attempt to be exhaustive but try instead to give some personal interpretations and some research directions. We discuss the notion of numeration systems, recognizable sets of integers and automatic sequences. We briefly sketch some results about transcendence related to the representation of real numbers. We conclude with some applications to combinatorial game theory and verification of infinite-state systems and present a list of open problems.
\end{abstract}

\section{Introduction}

It is challenging to give a talk about the interactions existing between formal language theory and number theory. The topic is vast, has several entry points and many applications. To cite just a few: non-adjacent form (NAF) representations to speed up computations arising in elliptic curve cryptography 61, verification of infinite-state systems [23], combinatorial game theory, fractals and tilings 82 20, transcendence results, dynamical systems and ergodic theory [19. Chap. 5-7], 1373. For instance, there exist tight and fruitful links between properties sought for in dynamical systems and combinatorial properties of the corresponding words and languages.

The aim of this paper is to briefly survey some results mostly emerging from the seminal papers of Cobham of the late sixties and early seventies [35|36 37, while also trying to give some personal interpretations and some research directions. We do not provide an exhaustive survey of the existing literature but we will give some pointers that we hope could be useful to the reader.

When one considers such interactions, the main ingredient is definitely the notion of numeration system, which provides a bridge between a set of numbers (integers, real numbers or elements of some other algebraic structures 689]) and formal language theory. On the one hand, arithmetic properties of numbers or sets of numbers are of interest and on the other hand, syntactical properties of the corresponding representations may be studied. To start with, we consider the familiar integer base $k \geq 2$ numeration system. Any integer $n>0$ is uniquely represented by a finite word (its $k$-ary representation) $\operatorname{rep}_{k}(n)=d_{\ell} \cdots d_{0}$ over

\footnotetext{
* Dedicated to the memory of my grandfather Georges Henderyckx 1930-2010.
} 
the alphabet $A_{k}=\{0, \ldots, k-1\}$ such that $\sum_{i=0}^{\ell} d_{i} k^{i}=n$ and $d_{\ell} \neq 0$. Note that imposing the uniqueness of the representation allows us to define a map $\operatorname{rep}_{k}: \mathbb{N} \rightarrow A_{k}^{*}$. Nevertheless, in many contexts it is useful to consider all the representations of an integer $n$ over a given finite alphabet $D \subset \mathbb{Z}$, that is all the words $c_{\ell} \cdots c_{0} \in D^{*}$ such that $\sum_{i=0}^{\ell} c_{i} k^{i}=n$. For instance, if $w$ is the $k$-ary representation of $n$ and if the alphabet $D$ is simply $A_{k}$, then for all $j>0,0^{j} w$ is another representation of $n$.

In the same way, any real number $r \in(0,1)$ is represented by an infinite word $d_{1} d_{2} \cdots$ over $A_{k}$ such that $\sum_{i=1}^{+\infty} d_{i} k^{-i}=r$. Uniqueness of the representation may be obtained by taking the maximal word for the lexicographic ordering on $A_{k}^{\omega}$ satisfying the latter equality; in this case, the sequence $\left(d_{i}\right)_{i \geq 1}$ is not ultimately constant and equal to $k-1$ : there is no $N$ such that, for all $n \geq N$, $d_{n}=k-1$. Therefore, to represent a real number $r>0$, we take separately its integer part $\lfloor r\rfloor$ and its fractional part $\{r\}$. Base $k$-complements or signed number representations [70] can be used to represent negative elements as well, the sign being determined by the most significant digit which is thus 0 or $k-1$. By convention, the empty word $\varepsilon$ represents 0 , i.e., $\operatorname{rep}_{k}(0)=\varepsilon$. If the numeration system is fixed, say the base $k$ is given, then any integer $n$ (resp. any real number $r>0$ ) corresponds to a finite (resp. infinite) word over $A_{k}$ (resp. over $A_{k} \cup\{\star\}$, where $\star$ is a new symbol used as a separator). Therefore any set of numbers corresponds to a language of representations and we naturally seek for some link between the arithmetic properties of the numbers belonging to the set and the syntactical properties of the corresponding representations. Let $X$ be a subset of $\mathbb{N}$. Having in mind Chomsky's hierarchy, the set $X$ could be considered quite "simple" from an algorithmic point of view whenever the set of $k$-ary representations of the elements in $X$ is a regular (or rational) language accepted by a finite automaton. A set $X \subseteq \mathbb{N}$ satisfying this property is said to be $k$ recognizable. Note that $X$ is $k$-recognizable if and only if $0^{*} \operatorname{rep}_{k}(X)$ is regular. As an example, a DFA (i.e., a deterministic finite automaton) accepting exactly the binary representations of the integers congruent to $3(\bmod 4)$ is given in Figure 1. Similarly, a set $X \subseteq \mathbb{R}$ of real numbers is $k$-recognizable if there exists

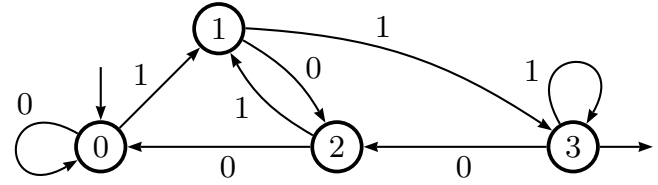

Fig. 1. A finite automaton accepting $0^{*} \operatorname{rep}_{2}(4 \mathbb{N}+3)$.

a finite (non-deterministic) Büchi automaton accepting all the $k$-representations over $A_{k}$ of the elements in $X$, that is, the representations starting with an arbitrary number of leading zeroes, and in particular the ones ending with $(k-1)^{\omega}$. Such an automaton is often called a Real Number Automaton [25. The Büchi automaton in Figure 2 (borrowed from a talk given by V. Bruyère) accepts all the possible binary encodings (using base 2-complement for negative numbers) 
of elements in the set $\{2 n+(0,4 / 3) \mid n \in \mathbb{Z}\}$. For instance $3 / 2$ is encoded by the language of infinite words $0^{+} 1 \star 10^{\omega} \cup 0^{+} 1 \star 01^{\omega}$. Note that the transition $3 \stackrel{\star}{\longrightarrow} 6$ (resp. $2 \stackrel{\star}{\longrightarrow} 4$ ) is considered for an odd (resp. even) integer part and the series $\sum_{i=1}^{+\infty} 4^{-i}=1 / 3$ corresponds to the cycle $\{5,6\}$.

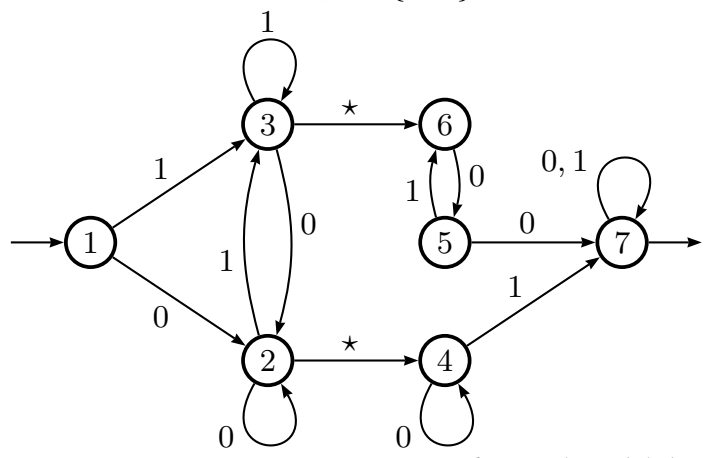

Fig. 2. A Büchi automaton accepting $\{2 n+(0,4 / 3) \mid n \in \mathbb{Z}\}$.

To generalize the $k$-ary integer base system, it is quite natural to consider an increasing sequence of integers, like the Fibonacci sequence, as a numeration basis to get a greedy decomposition of any integer (see Definition 2) or the negative powers of a real number $\beta>1$ to develop any real $r \in(0,1)$ as $\sum_{i=1}^{+\infty} c_{i} \beta^{-i}$ with the coefficients $c_{i}$ belonging to a convenient finite alphabet. Let us point out Fraenkel's paper [54 for some general ideas about representations of integers in various numeration systems. Among non-standard decompositions of integers, let us mention the so-called combinatorial numeration system going back to Lehmer and Katona, where integers are decomposed using binomial coefficients with some prescribed property, also see [33], and the factorial numeration system [72]. In Frougny and Sakarovitch's chapter [19, Chap. 2] and in Frougny's chapter [76, Chap. 7] many details on recognizable sets and about the representation of integers and real numbers are given. In particular, Parry's $\beta$-developments of real numbers [80] are presented in the latter reference. Abstract numeration systems (see Definition [6) are a general framework to study recognizable sets of integers, see [71] and [19, Chap. 3].

The seminal work of Cobham may be considered as a starting point for the study of recognizable sets for at least three reasons. Let us sketch these below. Details and definitions will be given in the next sections.

(i) Cobham's theorem from 1969 [36] states that the recognizability of a set of integers, as introduced above, strongly depends on the choice of the base, e.g., there are sets which are 2-recognizable but not 3-recognizable. The only subsets of $\mathbb{N}$ that are recognizable in all bases are exactly the ultimately periodic sets, i.e., the finite unions of arithmetic progressions. See Theorem 1 in Section 2 below for the exact statement of the result. It is an easy exercise to show that an arithmetic progression is $k$-recognizable for all $k \geq 2$ (e.g., Figure 11). See for instance [85, prologue] about the machine à diviser de 
Blaise Pascal. In that direction, an interesting question [7] is to obtain the state complexity of the minimal automaton recognizing a given divisibility criterion in an integer base. For this state complexity question studied in the wider context of linear numeration systems (cf. Definition 31), see [32].

The base dependence of recognizability shown by Cobham's result strongly motivates the general study of recognizable sets and the introduction of non-standard or exotic numeration systems based on an increasing sequence satisfying a linear recurrence relation.

For integer base $k$ numeration systems, nice characterizations of recognizable sets are well-known: logical characterization by first order formulas in a suitable extension of the Presburger arithmetic $\langle\mathbb{N},+\rangle, k$-automatic characteristic sequence generated through a uniform morphism of length $k$, characterization in terms of algebraic formal power series for a prime base. See the excellent survey [29] where the so-called Cobham-Semenov' theorem, which extends Cobham's original result from 1969 to subsets of $\mathbb{N}^{d}, d \geq 2$, is also presented. Recall that the characteristic sequence $\left(x_{i}\right)_{i \geq 0} \in\{0,1\}^{\mathbb{N}}$ of $X \subseteq \mathbb{N}$ is defined by $x_{i}=1$ if and only if $i \in X$. It is not our goal to give here a full list of pointers to the existing bibliography on the ramifications of Cobham's theorem, see for instance [4]. For presentations of Cobham's theorem based on Georges Hansel's work, see 81/12] together with 84].

(ii) The next paper of Cobham from 1972 37] introduced the concept of $k$ automatic sequences (originally called tag sequences, see Definition 5) and linked numeration systems with the so-called substitutions and morphic words (see Definition (4). It is easy to see that a set $X \subseteq \mathbb{N}$ is $k$-recognizable if and only if the characteristic sequence of $X$ is a $k$-automatic infinite word over $\{0,1\}$. For a comprehensive book on $k$-automatic sequences, see [12. As we will see, this approach gives another way to generalize the notion of a recognizable set by considering sets having a morphic characteristic sequence (see Remark 2). Details will be presented in Section 3 .

(iii) As the reader may already have noticed, this survey is mainly oriented towards sets of numbers (integers) giving rise to a language of representations. Another direction should be to consider a single real number and the infinite word representing it in a given base. Alan Cobham also conjectured the following result proved later on by Adamczewski and Bugeaud. Let $\alpha$ be an algebraic irrational real number. Then the base- $k$ expansion of $\alpha$ cannot be generated by a finite automaton. Cobham's question follows a question of Hartmanis and Stearns 64]: does it exist an algebraic irrational number computable in linear time by a (multi-tape) Turing machine? In the same way, if an infinite word $w$ over the finite alphabet $A_{k}$ of digits has some specific combinatorial properties (like, a low factor complexity, or being morphic or substitutive), is the corresponding real number having $w$ as $k$-ary representation transcendental? Let us mention that several surveys in that direction are worth of reading [77, Chap. 10], [19, Chap. 8], 292. We will briefly sketch some of these developments in Section 4 . 
In Section [5, we sketch some of the links existing between numeration systems, combinatorics on words and combinatorial game theory. Cobham's theorem about base dependence also appears in the framework of the verification of infinite state systems, see Section 6. Finally, in Section 7 we give some paths to the literature that the interested reader may follow and in Section 8, we present some open questions.

\section{Cobham's Theorem and Base Dependence}

Two integers $k, \ell \geq 2$ are multiplicatively independent if the only integers $m, n$ such that $k^{m}=\ell^{n}$ are $m=n=0$. Otherwise stated, $k, \ell \geq 2$ are multiplicatively independent if and only if $\log k / \log \ell$ is irrational. Recall a classical result in elementary number theory, known as Kronecker's theorem: if $\theta>0$ is irrational, then the set $\{\{n \theta\} \mid n>0\}$ is dense in $[0,1]$. Such result is an important argument in the proof of the following result.

Theorem 1 (Cobham's theorem [36]). Let $k, \ell \geq 2$ be two multiplicatively independent integers. $A$ set $X \subseteq \mathbb{N}$ is simultaneously $k$-recognizable and $\ell$ recognizable if and only if $X$ is ultimately periodic.

Obviously the set $P_{2}=\left\{2^{n} \mid n \geq 1\right\}$ of powers of two is 2-recognizable because $\operatorname{rep}_{2}\left(P_{2}\right)=10^{*}$. But since $P_{2}$ is not ultimately periodic, Cobham's theorem implies that $P_{2}$ cannot be 3 -recognizable. To see that a given infinite ordered set $X=\left\{x_{0}<x_{1}<x_{2}<\cdots\right\}$ is $k$-recognizable for no base $k \geq 2$ at all, we can use results like the following one where the behavior of the ratio (resp. difference) of any two consecutive elements in $X$ is studied through the quantities

$$
\mathbf{R}_{X}:=\limsup _{i \rightarrow \infty} \frac{x_{i+1}}{x_{i}} \text { and } \mathbf{D}_{X}:=\limsup _{i \rightarrow \infty}\left(x_{i+1}-x_{i}\right) .
$$

Theorem 2 (Gap theorem [37]). Let $k \geq 2$. If $X \subseteq \mathbb{N}$ is a k-recognizable infinite subset of $\mathbb{N}$, then either $\mathbf{R}_{X}>1$ or $\mathbf{D}_{X}<+\infty$.

Corollary 1. Let $a \in \mathbb{N}_{\geq 2}$. The set of primes and the set $\left\{n^{a} \mid n \geq 0\right\}$ are never $k$-recognizable for any integer base $k \geq 2$.

Proofs of the Gap theorem and its corollary can also be found in [50. It is easy to show that $X \subseteq \mathbb{N}$ is $k$-recognizable if and only if it is $k^{n}$-recognizable, $n \in \mathbb{N} \backslash\{0\}$. As a consequence of Cobham's theorem, sets of integers can be classified into three categories:

- ultimately periodic sets which are recognizable for all bases,

- sets which are $k$-recognizable for some $k \geq 2$, and which are $\ell$-recognizable only for those $\ell \geq 2$ such that $k$ and $\ell$ are multiplicatively dependent bases, for example, the set $P_{2}$ of powers of two,

- sets which are $k$-recognizable for no base $k \geq 2$ at all, for example, the set of squares. 
Definition 1. An infinite ordered set $X=\left\{x_{0}<x_{1}<x_{2}<\cdots\right\}$ such that $\mathbf{D}_{X}<+\infty$ is said to be syndetic or with bounded gaps. Otherwise stated, $X$ is syndetic if there exists $C>0$ such that, for all $n \geq 0, x_{n+1}-x_{n}<C$.

If $X \subseteq \mathbb{N}$ is ultimately periodic, then $X$ is syndetic. Note that the converse does not hold. For instance, consider the complement of the set $\left\{2^{n} \mid n \geq 0\right\}$ which is syndetic, 2-recognizable but not ultimately periodic.

Example 1 (Thue-Morse set). Let $n \in \mathbb{N}$. Denote by $s_{k}(n)$ the classical numbertheoretic function summing up the digits appearing in $\operatorname{rep}_{k}(n)$. As a classical example, consider the set $T=\left\{n \in \mathbb{N} \mid s_{2}(n) \equiv 0 \bmod 2\right\}$. This set is 2-recognizable and syndetic but not ultimately periodic. It appears in several contexts [11] and in particular, it provides a solution to Prouhet's problem (also known as the Prouhet-Tarry-Escott problem which is a special case of a multi-grade equation).

The set of squares is $k$-recognizable for no integer base $k$ but as we shall see this set is recognizable for some non-standard numeration systems (see Example (4). One possible extension of $k$-ary numeration systems is to consider a numeration basis.

Definition 2. A numeration basis is an increasing sequence $U=\left(U_{n}\right)_{n \geq 0}$ of integers such that $U_{0}=1$ and $\sup _{i \geq 0} U_{i+1} / U_{i}$ is bounded.

Using the greedy algorithm, any integer $n>0$ has a unique decomposition

$$
n=\sum_{i=0}^{\ell} c_{i} U_{i}
$$

where the coefficients $c_{i}$ belong to the finite set $A_{U}=\left\{0, \ldots, \sup \left\lceil U_{i+1} / U_{i}\right\rceil-1\right\}$. Indeed there exists a unique $\ell \geq 0$ such that $U_{\ell} \leq n<U_{\ell+1}$. Set $r_{\ell}=n$. For all $i=\ell, \ldots, 1$, proceed to the Euclidean division $r_{i}=c_{i} U_{i}+r_{i-1}$, with $r_{i-1}<U_{i}$. The word $c_{\ell} \cdots c_{0}$ is the (normal) $U$-representation of $n$ and is denoted by $\operatorname{rep}_{U}(n)$. Naturally, these non-standard numeration systems include the usual integer base $k$ system by taking $U_{n}=k^{n}$ for all $n \geq 0$. The numerical value map $\operatorname{val}_{U}: A_{U}^{*} \rightarrow \mathbb{N}$ maps any word $d_{\ell} \cdots d_{0}$ over $A_{U}$ onto $\sum_{i=0}^{\ell} d_{i} U_{i}$.

Remark 1. By contrast with abstract numeration systems that will be introduced later on, when dealing with a numeration basis we often use the terminology of a positional numeration system to emphasize the fact that a digit $d \in A_{U}$ in the $i$ th position (counting from the right, i.e., considering the least significant digit first) of a $U$-representation has a weight $d$ multiplied by the corresponding element $U_{i}$ of the basis.

Having in mind the notion of $k$-recognizable sets, a set $X \subseteq \mathbb{N}$ is said to be $U$-recognizable if $\operatorname{rep}_{U}(X)=\left\{\operatorname{rep}_{U}(n) \mid n \in X\right\}$ is a regular language over the alphabet $A_{U}$. Note that $\operatorname{rep}_{U}(X)$ is regular if and only if $0^{*} \operatorname{rep}_{U}(X)$ is regular. 
Definition 3. A numeration basis $U=\left(U_{n}\right)_{n \geq 0}$ is said to be linear, if there exist $a_{0}, \ldots, a_{k-1} \in \mathbb{Z}$ such that

$$
\forall n \geq 0, U_{n+k}=a_{k-1} U_{n+k-1}+\cdots+a_{0} U_{n} .
$$

If $\lim _{n \rightarrow \infty} U_{n+1} / U_{n}=\beta$ for some real $\beta>1$, then $U$ is said to satisfy the dominant root condition and $\beta$ is called the dominant root of the recurrence.

If $\mathbb{N}$ is $U$-recognizable, then $U$ is a linear numeration basis 8919] (hint: observe that $\left.\operatorname{rep}_{U}\left(\left\{U_{n} \mid n \geq 0\right\}\right)=10^{*}\right)$. For a discussion on sufficient conditions on the recurrence relation satisfied by $U$ for the $U$-recognizability of $\mathbb{N}$, see 65. and [75]. In particular, as shown by the next result, for a linear numeration basis $U$, the set $\mathbb{N}$ is $U$-recognizable if and only if all ultimately periodic sets are $U$-recognizable.

Theorem 3 (Folklore [19]). Let $p, r \geq 0$. If $U=\left(U_{n}\right)_{n \geq 0}$ is a linear numeration basis, then

$$
\operatorname{val}_{U}^{-1}(p \mathbb{N}+r)=\left\{c_{\ell} \cdots c_{0} \in A_{U}^{*} \mid \sum_{k=0}^{\ell} c_{k} U_{k} \in p \mathbb{N}+r\right\}
$$

is accepted by a DFA that can be effectively constructed. In particular, if $\mathbb{N}$ is $U$-recognizable, then any ultimately periodic set is U-recognizable.

Example 2. Consider the Fibonacci numeration system given by the basis $F_{0}=$ $1, F_{1}=2$ and $F_{n+2}=F_{n+1}+F_{n}$ for all $n \geq 0$. For this system, $0^{*} \operatorname{rep}_{F}(\mathbb{N})$ is given by the set of words over $\{0,1\}$ avoiding the factor 11 and the set of even numbers is $U$-recognizable 32] using the DFA shown in Figure 3 .

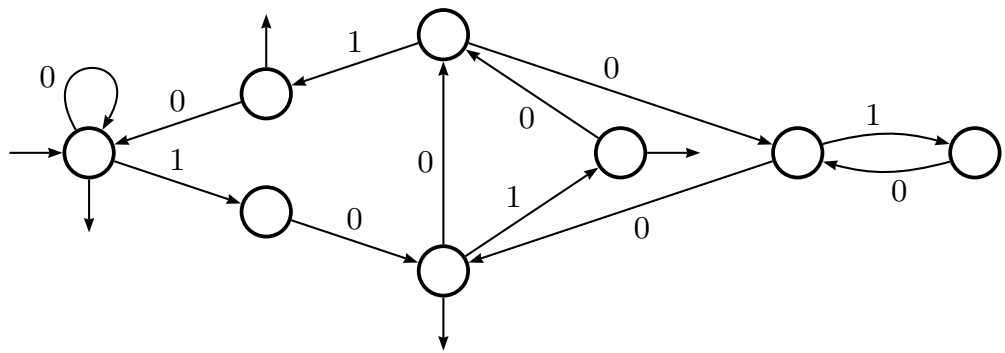

Fig. 3. A finite automaton accepting $0^{*} \operatorname{rep}_{F}(2 \mathbb{N})$

To conclude this section, we present a linear numeration basis $U$ such that the set of squares $\mathcal{Q}=\left\{n^{2} \mid n \in \mathbb{N}\right\}$ is $U$-recognizable. This set will also be used in Example 4 to get a set having a morphic characteristic sequence.

Example 3. Consider the sequence given $U_{n}=(n+1)^{2}$ for all $n \geq 0$. This sequence satisfies, for all $n \geq 0$, the relation $U_{n+3}=3 U_{n+2}-3 U_{n+1}+U_{n}$. In that case, $\operatorname{rep}_{U}(\mathbb{N}) \cap 10^{*} 10^{*}=\left\{10^{a} 10^{b} \mid b^{2}<2 a+4\right\}$ showing with the pumping lemma that $\mathbb{N}$ is not $U$-recognizable $\left[89\right.$. But trivially, we have $\operatorname{rep}_{U}(\mathcal{Q})=10^{*}$. 


\section{Substitutions and Abstract Numeration Systems}

For basic facts on morphisms over $A^{*}$ or the usual distance put on $A^{\omega}$ (which gives a notion of convergence), see classic textbooks like [12 19 76]. Let $A$ be a finite alphabet and $\sigma: A^{*} \rightarrow A^{*}$ be a morphism. If there exist a letter $a \in A$ and a word $u \in A^{+}$such that $\sigma(a)=a u$ and moreover, if $\lim _{n \rightarrow+\infty}\left|\sigma^{n}(a)\right|=+\infty$, then $\sigma$ is said to be prolongable on $a$.

Definition 4. Let $\sigma: A^{*} \rightarrow A^{*}$ be a morphism prolongable on a. We have

$$
\sigma(a)=a u, \sigma^{2}(a)=a u \sigma(u), \sigma^{3}(a)=a u \sigma(u) \sigma^{2}(u), \ldots .
$$

Since, for all $n \in \mathbb{N}, \sigma^{n}(a)$ is a prefix of $\sigma^{n+1}(a)$ and because $\left|\sigma^{n}(a)\right|$ tends to infinity when $n \rightarrow+\infty$, the sequence $\left(\sigma^{n}(a)\right)_{n \geq 0}$ converges to an infinite word denoted by $\sigma^{\omega}(a)$ and given by

$$
\sigma^{\omega}(a):=\lim _{n \rightarrow+\infty} \sigma^{n}(a)=a u \sigma(u) \sigma^{2}(u) \sigma^{3}(u) \cdots
$$

This infinite word is a fixed point of $\sigma$. An infinite word obtained in this way by iterating a prolongable morphism is said to be purely morphic. In the literature, one also finds the term pure morphic. If $x \in A^{\mathbb{N}}$ is purely morphic and if $\tau$ : $A \rightarrow B$ is a coding (or letter-to-letter morphism), then the word $y=\tau(x)$ is said to be morphic.

Definition 5. Let $k \geq 2$. A morphic word $w \in B^{\omega}$ is $k$-automatic if there exists a morphism $\sigma: A^{*} \rightarrow A^{*}$ and a coding $\tau$ such that $w=\tau\left(\sigma^{\omega}(a)\right)$ and, for all $c \in A,|\sigma(c)|=k$. A morphism satisfying this latter property is said to be uniform.

The link between $k$-recognizable sets and $k$-automatic sequences is given by the following result. In particular, in the proof of this result, it is interesting to note that an automaton is canonically associated with a morphism.

Theorem 4. 37 An infinite word $w=w_{0} w_{1} w_{2} \cdots$ over an alphabet $A$ is $k$ automatic if and only if, for all $a \in A$, the set $X_{a}=\left\{i \in \mathbb{N} \mid w_{i}=a\right\}$ is $k$-recognizable.

Otherwise stated, $w=w_{0} w_{1} w_{2} \cdots \in A^{\omega}$ is $k$-automatic if and only if there exists a deterministic finite automaton with output (DFAO) $\mathcal{M}$ where $Q$ is the set of states of $\mathcal{M}, \delta: Q \times A_{k} \rightarrow Q$ (resp. $\tau: Q \rightarrow A$ ) is the transition function (resp. output function) of $\mathcal{M}$, such that $\tau\left(\delta\left(q_{0}, \operatorname{rep}_{k}(n)\right)=w_{n}\right.$ for all $n \geq 0$.

Remark 2. Using automata as a model of computation, $U$-recognizable sets naturally raise some interest. On the same level, sets of integers having a morphic characteristic sequence can be considered as another natural generalization of the concept of $k$-recognizability. Iterations of a morphism may be used to get inductively further elements of the set defined by the morphism and a coding. As will be shown by Theorem 6] similarly to the case of uniform morphisms (as given in Definition [5] described above, the computation of a given element can also be done by using a DFAO and representations of integers in an abstract numeration system. 
Example 4. Consider the alphabet $A=\{a, b, c\}$ and the morphism $\sigma: A^{*} \rightarrow A^{*}$ defined by $\sigma: a \mapsto a b c c, b \mapsto b c c, c \mapsto c$. We get

$$
\sigma^{\omega}(a)=a b c c b c c c c b c c c c c c b c c c c c c c c b c c c c c c c c c c b c c \cdots .
$$

It is easy to see that considering the coding $\tau: a, b \mapsto 1$ and $\tau: c \mapsto 0$, the word $\tau\left(\sigma^{\omega}(a)\right)$ is the characteristic sequence of the set of squares.

The factor complexity of an infinite word $w$ is the non-decreasing function $p_{w}: \mathbb{N} \rightarrow \mathbb{N}$ mapping $n$ onto the number of distinct factors (or subwords) occurring in $w$. See for instance [19, Chap. 4]. For a survey on the factor complexity of morphic words, see [8]. In 1972, Cobham already observed that if $w$ is $k$-automatic, then $p_{w}$ is in $\mathcal{O}(n)$. For instance, the factor complexity of the characteristic sequence of the Thue-Morse set $T$ considered in Example 1 is computed in 27/39.

Theorem 5 (Morse-Hedlund's Theorem). Let $x=x_{0} x_{1} x_{2} \cdots$ be an infinite word over $A$. The following conditions are equivalent.

- The complexity function $p_{x}$ is bounded by a constant, i.e., there exists $C$ such that for all $n \in \mathbb{N}, p_{x}(n) \leq C$.

- There exists $N_{0} \in \mathbb{N}$ such that for all $n \geq N_{0}, p_{x}(n)=p_{x}\left(N_{0}\right)$.

- There exists $N_{0} \in \mathbb{N}$ such that $p_{x}\left(N_{0}\right)=N_{0}$.

- There exists $m \in \mathbb{N}$ such that $p_{x}(m)=p_{x}(m+1)$.

- The word $x$ is ultimately periodic.

In particular, non ultimately periodic sequences with low complexity are the socalled Sturmian sequences whose factor complexity is $p(n)=n+1$ for all $n \geq 1$. Note that such sequences are over a binary alphabet, $p(1)=2$. For a survey on Sturmian words, see for instance [76. Since Pansiot's work [79, the factor complexity of a non ultimately periodic purely morphic word $w$ is well-known, see for instance [19, Chap. 4] or the survey [8, there exists constants $C_{1}, C_{2}$ such that $C_{1} f(n) \leq p_{w}(n) \leq C_{2} f(n)$ where $f(n) \in\left\{n, n \log n, n \log \log n, n^{2}\right\}$.

Remark 3. F. Durand has achieved a lot of work towards a general version of Cobham's theorem for morphic words 45/46 47. Without giving much details (see for instance 48 for a detailed account), with a non-erasing morphism $\sigma$ over $A=\left\{a_{1}, \ldots, a_{t}\right\}$ (i.e., $\sigma\left(\sigma_{i}\right) \neq \varepsilon$ for all $i$ ) generating a morphic word $w$ (also using an extra coding) is associated a matrix $\mathbf{M}_{\sigma}$ (like the adjacency matrix of a graph) where, for all $i, j,\left(\mathbf{M}_{\sigma}\right)_{i, j}$ is the number of occurrences of the letter $a_{i}$ in the image $\sigma\left(a_{j}\right)$. Considering the morphism in Example 4, we get

$$
\mathbf{M}_{\sigma}=\left(\begin{array}{lll}
1 & 0 & 0 \\
1 & 1 & 0 \\
2 & 2 & 1
\end{array}\right)
$$

Then considering the irreducible components (i.e., the strongly connected components of the associated automaton) of the matrix $\mathbf{M}_{\sigma}$ and the theorem of 
Perron-Frobenius, a real number $\beta>0$ is associated with the morphism. The word $w$ is therefore said to be $\beta$-substitutive. Let $\alpha, \beta>1$ be two multiplicatively independent Perron numbers (the notion of multiplicative independence extends to real numbers $>1$ ). Under some mild assumptions [48, if $w$ is both $\alpha$-substitutive and $\beta$-substitutive, then it is ultimately periodic. It is a natural generalization of the fact that if $k, \ell \geq 2$ are multiplicatively independent, then a word which is both $k$-automatic and $\ell$-automatic is ultimately periodic.

Example 5. The consecrated Fibonacci word, i.e., the unique fixed point of $\sigma: 0 \mapsto 01,1 \mapsto 0$, is $\alpha$-substitutive where $\alpha$ is the Golden ratio $(1+\sqrt{5}) / 2$. Therefore, this infinite word is $k$-automatic for no integer $k \geq 2$. Indeed, $k$ and the Golden ratio are multiplicatively independent.

In view of Theorem 3, it is desirable for a numeration basis $U$ that the set $\mathbb{N}$ be $U$-recognizable. In that case, one can use a finite automaton to test whether or not a given word over $A_{U}$ is a valid $U$-representation. Taking this requirement as a basic assumption and observing that for all integers $x, y$, we have $x<y$ if and only if $\operatorname{rep}_{U}(x)$ is genealogically less than $\operatorname{rep}_{U}(y)$, we introduce the concept of an abstract numeration system. To define the genealogical order (also called radix or military order), first order words by increasing length and for words of the same length, take the usual lexicographical order induced by the ordering of the alphabet.

Definition 6. Let $L$ be an infinite regular language over a totally ordered alphabet $(A,<)$. An abstract numeration system is the triple $S=(L, A,<)$. Ordering by increasing genealogical order the words in $L$ provides a one-to-one correspondence between $L$ and $\mathbb{N}$. The nth word in $L$ (starting from 0 ) is denoted by $\operatorname{rep}_{S}(n)$ and the inverse map $\operatorname{val}_{S}: L \rightarrow \mathbb{N}$ is such that $\operatorname{val}_{S}\left(\operatorname{rep}_{S}(n)\right)=n$. Any numeration basis $U$ such that $\mathbb{N}$ is $U$-recognizable is a particular case of an abstract numeration system. In this respect, a set $X \subseteq \mathbb{N}$ is $S$-recognizable, if $\operatorname{rep}_{S}(X)$ is a regular language.

A sequence $w=w_{0} w_{1} \cdots$ is $S$-automatic if there exists a DFAO $\mathcal{M}$ where $\delta: Q \times A_{k} \rightarrow Q$ (resp. $\tau: Q \rightarrow A$ ) is the transition function (resp. output function) of $\mathcal{M}$, such that $\tau\left(\delta\left(q_{0}, \operatorname{rep}_{S}(n)\right)=w_{n}\right.$ for all $n \geq 0$.

Example 6. Again the set of squares $\mathcal{Q}$ is $S$-recognizable for the abstract numeration system $S=\left(a^{*} b^{*} \cup a^{*} c^{*},\{a, b, c\}, a<b<c\right)$. Indeed, we have

$$
a^{*} b^{*} \cup a^{*} c^{*}=\varepsilon, a, b, c, a a, a b, a c, b b, c c, a a a, \ldots
$$

and one can check that $\operatorname{rep}_{S}(\mathcal{Q})=a^{*}$ because the growth function of the language is $\#\left(\left(a^{*} b^{*} \cup a^{*} c^{*}\right) \cap\{a, b, c\}^{n}\right)=2 n+1$.

Theorem 4 can be generalized as follows [83, or [19, Ch. 3].

Theorem 6. An infinite word $w=w_{0} w_{1} w_{2} \cdots$ over an alphabet $A$ is morphic if and only if there exists an abstract numeration system $S$ such that $w$ is $S$ automatic. 
Note that for generalization of Theorems 4 and 6 to a multidimensional setting, see Salon's work 86 87] and 31] respectively. Moreover, thanks to the above result, Durand's work can also to some extent be expressed in terms of abstract numeration systems. Observe that in Example 6 the abstract numeration system is based on a regular language having a polynomial growth. This corresponds to the case where the dominating eigenvalue of the matrix associated with the morphism is 1 . Such a situation (polynomial versus exponential growth) is considered in [49. Indeed, note that in the discussion about a morphic version of Durand-Cobham's theorem in Remark 3 we only considered morphisms with exponential growth, i.e., the dominating eigenvalue being $>1$.

\section{Transcendental Numbers}

This short section is based on a lecture given by B. Adamczewski during the last CANT summer school in Liège [19, Chap. 8] and on [92. We also refer the reader to 2. It illustrates one of the strong links existing between combinatorics on words and number theory. For a survey on combinatorics on words, see for instance [17/34. Recall that a complex number which is a root of a non-zero polynomial with rational (or equivalently integer) coefficients is said to be algebraic. Otherwise, it is said to be transcendental. Since Borel's work, one thinks that base- $k$ expansion of algebraic irrational numbers are "complex" and not much is known about their properties.

With any infinite word $w=w_{1} w_{2} \cdots$ over the alphabet of digits $A_{k}=$ $\{0, \ldots, k-1\}$ is associated the real number $\sum_{i=1}^{+\infty} w_{i} k^{-i}$ in $[0,1]$. Clearly, a real number $\alpha$ is algebraic (over $\mathbb{Q}$ ) if and only if, for all $z \in \mathbb{Z}, \alpha+z$ is algebraic. Indeed, if $\alpha$ is a root of the polynomial $P(X) \in \mathbb{Q}(X)$, then $\alpha+z$ is a root of $P(X-z) \in \mathbb{Q}(X)$. Hence, we can restrict ourselves to numbers in $(0,1)$.

Transcendence of a number whose binary expansion is Sturmian has been proved in 1997 [51].

Example 7. Consider again the Fibonacci word $f=f_{1} f_{2} f_{3} \cdots=010010 \cdots$. The real number $\sum_{i=1}^{+\infty} f_{i} 2^{-i}$ is transcendental.

Let $k \in \mathbb{N} \backslash\{0,1\}$. The factor complexity of the $k$-ary expansion $w$ of every irrational algebraic number satisfies

$$
\liminf _{n \rightarrow \infty}\left(p_{w}(n)-n\right)=+\infty .
$$

The main tool is a $p$-adic version of the Thue-Siegel-Roth theorem due to Ridout.

A combinatorial transcendence criterion obtained in [4] using Schmidt's subspace theorem [88] is used to obtain the following result.

Theorem 7 (Adamczewski and Bugeaud [3]). Let $k \in \mathbb{N} \backslash\{0,1\}$. The factor complexity of the $k$-ary expansion $w$ of a real irrational algebraic number satisfies

$$
\lim _{n \rightarrow+\infty} \frac{p_{w}(n)}{n}=+\infty
$$


Let $k \geq 2$ be an integer. If $\alpha$ is a real irrational number whose $k$-ary expansion has factor complexity in $\mathcal{O}(n)$, then $\alpha$ is transcendental. Since, it is well-known [37] that automatic sequences have factor complexity $p(n) \in \mathcal{O}(n)$, we can deduce that if a real irrational number has an automatic $k$-ary expansion, then it is transcendental.

Theorem 8 (Bugeaud and Evertse [30]). Let $k \geq 2$ be an integer and $\xi$ be a real irrational algebraic number with $0<\xi<1$. Then for any real number $\eta<1 / 11$, the factor complexity $p(n)$ of the $k$-ary expansion of $\xi$ satisfies

$$
\lim _{n \rightarrow+\infty} \frac{p(n)}{n(\log n)^{\eta}}=+\infty .
$$

In [5], it is shown that the binary expansion of an algebraic number contains infinitely many occurrences of $7 / 3$-powers. Hence the binary expansion of an algebraic number contains infinitely many overlaps.

\section{Combinatorial Game Theory}

Numeration systems, number theory and therefore formal language theory also have interesting connections with combinatorial game theory. In classical textbooks like 63 15] allusion to the game of Nim is made. See 16 57] for background on two player combinatorial games: no chance, no hidden information, same options for the two players who play alternatively, .... In particular, in removal games, we are looking for a winning strategy which allows a player to consummate a win regardless of the moves chosen by the other player. If such a strategy exists for given initial conditions, it is therefore natural to ask about the algorithmic complexity of the computation of the winning strategy. A first question to answer is to determine the status $\mathcal{N}$ or $\mathcal{P}$ of a given position [57.

A $\mathcal{N}$-position, or winning position, is a position for which a winning strategy exists for the player who starts. A $\mathcal{P}$-position is a position for which all options lead to a $\mathcal{N}$-position, and is thus winning for the second playen 1 . In the game of Nim played on two piles of tokens, two players play alternatively and remove a positive number of tokens from one of the piles. The player removing the last token win. Otherwise stated, the first player unable to move loses (normal condition). In [12, connections between the game of Nim (values of the Sprague-Grundy function) and the notion of 2-regular functions in the sense of Allouche and Shallit is observed (finiteness of the 2-kernel). In the famous Wythoff's game, an extra move is allowed: removing the same positive number of tokens on both piles. The game of Nim can be easily generalized to $n$ piles of tokens contrarily to Wythoff's game where extensions have been presented but no suitable generalization is known: for the $\mathcal{P}$-positions playing with an odd

\footnotetext{
${ }^{1}$ In the game graph $\mathcal{G}$ where vertices are positions and directed edges are the allowed moves, the set of $\mathcal{P}$-positions is the kernel of $\mathcal{G}$ : there is no move between any two $\mathcal{P}$-positions and from any $\mathcal{N}$-position, there exists a move to a $\mathcal{P}$-position.
} 
number of piles is similar to the game of Nim and playing with an even number of piles is hard 44435352. In the last reference, Wythoff's game is considered as a Prime game. Informally, a game whose generalization to more than one or two piles seems to be very hard.

For instance, A. Fraenkel makes great use of various numeration systems to get characterizations of $\mathcal{P}$-positions [56]. As an example, in Wythoff's game, a position $(x, y)$ is a $\mathcal{P}$-position if and only if the $F$-representation $\operatorname{rep}_{F}(x)$ ends with an even number of zeroes and $\operatorname{rep}_{F}(y)=\operatorname{rep}_{F}(x) 0$ is the left shift of the first component, where $F$ is the numeration basis given by the Fibonacci sequence from Example 2 53. Similarly, $(x, y)$ is a $\mathcal{P}$-position if there exists $n$ such that $(x, y)=\left(\lfloor n \alpha\rfloor,\left\lfloor n \alpha^{2}\right\rfloor\right)$ where $\alpha$ is the Golden ratio. So complementary Beatty sequences also enter the picture of combinatorial games 42,53.38.

In 41] moves that can be adjoined without changing the set of $\mathcal{P}$-positions are characterized using the formalism of morphisms and the fact that the computation of the successor function in the Fibonacci system is realized by a finite transducer [59]. Let $a \in \mathbb{N} \backslash\{0,1\}$. In the parameterized version of Wythoff's game where a player can remove $k$ tokens from one pile and $\ell$ from the other [53], with the condition $|k-\ell|<a$, the Ostrowski numeration system [18] based on the convergents of a continued fraction is used.

It is interesting to note that obviously the $\mathcal{P}$-positions of Wythoff's game are also characterized by the Fibonacci word introduced in Example 7 . The $n$th $\mathcal{P}$-position is given by the pair of indices of the $n$th symbol 0 and $n$th symbol 1 occurring in the Fibonacci word. This simple observation relates combinatorial properties of morphic words like the Fibonacci or Tribonacci words to characterizations of $\mathcal{P}$-positions of games 44,4352. Morphic characterizations of $\mathcal{P}$-positions seems to recently raise some interest among combinatorial game theorists 52 .

\section{Applications for Verification of Infinite State Systems}

Sets of numbers recognized by finite automata arise when analyzing systems with unbounded mixed variables taking integer or real values. Therefore are considered systems such as timed or hybrid automata [21. One needs to develop data structures representing sets manipulated during the exploration of infinite state systems. For instance, it is often needed to compute the set of reachable configurations of such a system.

Let $k \geq 2$ be an integer. Recall that $\mathrm{A}$ set $X \subseteq \mathbb{R}$ is $k$-recognizable if there exists a Büchi automaton accepting all the $k$-representations of the elements in $X$. This notion extends to subsets of $\mathbb{R}^{d}$ and to Real Vector Automata or $R V A$. Also Büchi-Bruyère's Theorem giving a first order logical characterization of $k$-recognizable sets of integers holds in this context of real numbers for a suitable structure $\left\langle\mathbb{R}, \mathbb{Z},+, 0,<, V_{k}\right\rangle$, see 25 . Roughly speaking definability in $\langle\mathbb{R}, \mathbb{Z},+, 0,<\rangle$ of subsets of $\mathbb{R}^{d}$ is the natural extension of ultimately periodicity of subsets in $\mathbb{N}$. 
Theorem 9. 24. If a subset $X \subseteq \mathbb{R}^{d}$ is definable by a first-order formula in $\langle\mathbb{R}, \mathbb{Z},+, 0,<\rangle$, then $X$ written in base $k \geq 2$ is recognizable by a weak deterministic $R V A \mathcal{A}$.

Weakness means that each strongly connected component of $\mathcal{A}$ contains only accepting states or non-accepting states.

Theorem 10. 22 Let $k, \ell \geq 2$ be two multiplicatively independent integers. If $X \subseteq \mathbb{R}$ is both $k$-and $\ell$-recognizable by two weak deterministic $R V A$, then it is definable in $\langle\mathbb{R}, \mathbb{Z},+, 0,<\rangle$.

The extension of Cobham-Semenov's theorem for subsets of $\mathbb{R}^{d}$ in this setting is discussed in 23. The case of two coprime bases was first considered in 22]. Though written in a completely different language, a similar result was independently obtained in [1. This latter paper is motivated by the study of some fractal sets.

Remark 4. Weak deterministic RVA have a particular interest from an algorithmic point of view. They recognize languages that are recognizable by deterministic Büchi and deterministic co-Büchi automata. For instance, minimization algorithms in $\mathcal{O}(n \log n)$ exist for this class [74].

\section{Abridged Bibliographic Notes}

With a gentle introduction to the logical formalism, a good way to start with integer base numeration systems is to consider [29]. Each time I come back to this very well written survey, I learn something new. Then, it is a good idea to move to the "state of the art" linear numeration basis where the characteristic polynomial of the recurrence is the minimal polynomial of a Pisot number [28]. In parallel, one should consider Frougny's chapter [76, Chap. 7] and her very interesting work on the normalization map [58 and beta-expansions 60. As a good textbook on some of the aspects presented here, consider [12. The original paper of Cobham [37] is also worth of reading. For some general surveys on factor complexity and the Thue-Morse word, without any required background, see 8]11.

Then I cannot resist advertising [19] where in the spirit of Lothaire's series, we try to present the fruitful links existing between combinatorics on words, automata theory and number theory. It presents in a self-contained expository book much more material than is presented in this survey (ergodic theory, Rauzy fractal, joint spectral radius,...).

For a list of pointers on Cobham's theorem in various contexts, see 48 for an updated survey. Accounts of Perron-Frobenius theory can be found in many classical textbooks, but probably [73] is worth reading.

Connections between symbolic dynamics and formal language theory are fruitful: for the reader with no background in dynamics (for instance, no knowledge in measure theory is required) and on a very introductory level, consider [90. Then, move to the survey [13] and [82. 


\section{Some Open Problems}

We conclude with some general (and probably quite hard) open problems.

- As mentioned in Section 3, the most general version of Cobham's theorem still relies on some mild assumptions about the considered morphisms (details are not given in this survey). F. Durand refers to these as "good substitutions". One could hope to relax these hypotheses and still get the same result with full generality [4]. Up to now there is no proof of a Cobham-like theorem for a substitution having no main sub-substitution having the same dominating eigenvalue like $a \mapsto a a 0,0 \mapsto 01$ and $1 \mapsto 0$. In this latter example, the dominating eigenvalue is 2 but the substitution restricted to the alphabet $\{0,1\}$ has $(1+\sqrt{5}) / 2$ as dominating eigenvalue.

- Come back again to Cobham's theorem but this time for Gaussian integers $\mathbb{G}=\{a+i b \mid a, b \in \mathbb{Z}\}$. Indeed, these numbers have nice representations using the so-called canonical number systems [68. For canonical numeration systems in algebraic number fields, every integer has a unique finite expansion which is computed starting with the least significant digit first. A Cobham-like conjecture for Gaussian integers 62 is related to the famous Four Exponentials conjecture: let $\left\{\lambda_{1}, \lambda_{2}\right\}$ and $\left\{x_{1}, x_{2}\right\}$ be two pairs of rationally independent complex numbers. Then, one of the numbers $e^{\lambda_{1} x_{1}}, e^{\lambda_{1} x_{2}}$, $e^{\lambda_{2} x_{1}}, e^{\lambda_{2} x_{2}}$ is transcendental, for instance see [91].

- The philosophy of Cobham's theorem also appears when considering selfgenerating sets as introduced by Kimberling [69. For instance, consider the affine maps $f: \mathbb{N} \rightarrow \mathbb{N}, x \mapsto 2 x+1$ and $g: \mathbb{N} \rightarrow \mathbb{N}, x \mapsto 4 x+2$. A selfgenerating set obtained from $f$ and $g$ can be defined as the smallest subset $S$ of $\mathbb{N}$ containing 0 and such that $f(S) \subset S$ and $g(S) \subset S$. In our example, the first few elements in $S$ are

$0,1,2,3,5,6,7,10,11,13,14,15,21,23,26,27,29,30,31,42,43,47,53, \ldots$

One can therefore study the $k$-recognizability of $S$. If one considers maps where the multiplicative constants are multiplicatively independent, then Allouche, Shallit and Skordev conjectured that the corresponding set cannot be $k$-recognizable [10]. With some technical hypothesis about the multiplicative coefficients when there are at least three affine maps, this conjecture has been proved to be true in [67]. One could hope to prove this conjecture in full generality. A possible connection with smooth numbers (having only small prime factors in their decomposition) has been pointed out by J. Shallit.

- In combinatorial game theory the Sprague-Grundy function $g$ is of great interest. For instance, the positions for which $g$ vanishes are exactly the $\mathcal{P}$ position of the game and when considering sums of games (several games are played simultaneously and at each turn, the player chooses on which of those games he will made a move), it can be used to distinguish $\mathcal{N}$-positions [16]. For Wythoff's game, little is known about this function (see for instance [55]) even if its recursive definition is simple. The value of $g(x, y)$ is the minimum excluded value (Mex) of the set of $g(u, v)$ where $(u, v)$ is ranging 
amongst the options reachable from $(x, y)$. By definition Mex $\emptyset=0$ and $\operatorname{Mex} S=\min (\mathbb{N} \backslash S)$ for all finite set $S$.

\begin{tabular}{c|ccccccccccc} 
& 0 & 1 & 2 & 3 & 4 & 5 & 6 & 7 & 8 & 9 & $\cdots$ \\
\hline 0 & 0 & 1 & 2 & 3 & 4 & 5 & 6 & 7 & 8 & 9 & $\cdots$ \\
1 & 1 & 2 & 0 & 4 & 5 & 3 & 7 & 8 & 6 & 10 & \\
2 & 2 & 0 & 1 & 5 & 3 & 4 & 8 & 6 & 7 & 11 & \\
3 & 3 & 4 & 5 & 6 & 2 & 0 & 1 & 9 & 10 & 12 & \\
4 & 4 & 5 & 3 & 2 & 7 & 6 & 9 & 0 & 1 & 8 & \\
5 & 5 & 3 & 4 & 0 & 6 & 8 & 10 & 1 & 2 & 7 & \\
$\vdots$ & & & & & & & & & \\
$\vdots$ & & & & & & & & &
\end{tabular}

Let $F$ be the Fibonacci numeration basis. As suggested by the developments considered in 41] could the above infinite array reveal some morphic structure, like having a finite $F$-kernel where this set could be defined as the set of subarrays

$$
(g(x, y))_{\operatorname{rep}_{F}}(x) \in\{0,1\}^{*} u, \operatorname{rep}_{F}(y) \in\{0,1\}^{*} v
$$

for given suffixes $u, v$ ? For the generalization of $k$-kernel, see for instance 83 .

- Theorem [10 is a Cobham-like theorem for sets of real numbers, definability in $\langle\mathbb{R}, \mathbb{Z},+, 0,<\rangle$ being the counterpart to ultimate periodicity of a set of integers. Can a simpler proof of this result be achieved, for instance by considering the techniques developed in [1] Also could this result be extended to other kind of representations of real numbers. For instance, considering $\beta$-expansions of real numbers, we could define $\beta$-recognizable sets of real numbers and consider two multiplicatively independent real numbers $\alpha, \beta>1$. As a first step (and to mimic what has chronologically been done for sets of integers), one could consider a set of real numbers $X \subseteq \mathbb{R}$ which is both $k$-recognizable and $\beta$-recognizable by two weak deterministic RVA, with $k \geq 2$ an integer and $\beta$ a Pisot number like the Golden ratio, and ask is $X$ definable in $\langle\mathbb{R}, \mathbb{Z},+, 0,<\rangle$ ?

- About abstract numeration systems, several questions about $S$-recognizable sets are open. For instance, is there some reasonable logical characterization of the $S$-recognizable sets of integers which could be compared to the characterization in the extended Presburger arithmetic $\left\langle\mathbb{N},+, V_{k}\right\rangle$. But one can notice that in general, if $X$ and $Y$ are $S$-recognizable, there is no reason to have a $S$-recognizable set $X+Y$ (even when considering multiplication by a constant). Another question is to relate the growth function $n \mapsto \#\left(L \cap A^{n}\right)$ of the regular language $L$ on which the abstract numeration system $S$ is based and the $S$-recognizable set. For instance, if $P \in \mathbb{N}[X]$ is a polynomial such that $P(\mathbb{N})$ is $S$-recognizable, what can be said about the growth function of the language of numeration. Results like the one found in [14] could be of interest.

- Recently numeration systems based on the powers of a rational number have been introduced [6] (motivated by a number theoretic question from Mahler). 
These numerations reveal interesting and intriguing properties. For instance, little is known about the properties of the language of numeration $L_{3 / 2}$. For a given prefix $w$, compute the number of words of length $n$ in the quotient $w^{-1} L_{3 / 2}$.

- It is well-known since the work of Cobham 35 that a morphic infinite word $w=\tau\left(\sigma^{\omega}(a)\right.$ ) where $\sigma$ and $\tau$ are arbitrary morphisms (where both morphisms can be erasing and $\tau$ is not necessarily a coding) can be generated by a non-erasing morphism $\mu$ and a coding $\nu$. See for instance [12] for a comprehensive proof or 66 for an alternative presentation. All the known proofs rely on morphisms and are quite long: could one describe in the formalism of automata theory a somehow simpler proof?

- Let me also mention Hollander's conjecture when for a linear numeration basis $U$, the dominant root condition is not satisfied [65. He has conjectured that $\operatorname{rep}_{U}(\mathbb{N})$ can be regular only if there exists $n$ such that

$$
\lim _{j \rightarrow \infty} U_{j n+k} / U_{(j-1) n+k}
$$

exists and is independent of $k$, and the characteristic polynomial $p(X)$ of $U$ is such that $p(X)=q\left(X^{n}\right)$ where $q(X)$ is the minimal polynomial for a recurrence which gives a regular language [26].

- Let $p$ be a prime. Derksen proved that the zero set of a linear recurrence over a field of characteristic $p$ is $p$-automatic [402]. Could such a result and Cobham's theorem be used to get back the classical Skolem-Mahler-Lech theorem (the zero set of a linear recurrence over a field of characteristic 0 is ultimately periodic)?

- The reader fond of logic could also look back at the list of open problems given by Michaux and Villemaire [78. This survey paper is devoted to problems related to Büchi's characterization of sets of natural numbers recognizable by finite automata in base $k$, as well as to Cobham's and Semenov's extensions of it.

\section{Acknowledgments}

I would like to thank Boris Adamczewski, Valérie Berthé, Véronique Bruyère, Eric Duchêne, Narad Rampersad for the careful reading of a first draft of this paper. They provided me useful suggestions to improve this text.

\section{References}

1. B. Adamczewski and J. P. Bell. An analogue of Cobham's theorem for fractals. Trans. Amer. math. Soc. to appear.

2. B. Adamczewski and J. P. Bell. Automata in number theory. In J.-E. Pin, editor, Handbook, Automata: from Mathematics to Applications. Europ. Math. Soc. publishing house. to appear.

3. B. Adamczewski and Y. Bugeaud. On the complexity of algebraic numbers I: Expansion in integer bases. Ann. of Math., 165:547-565, 2007. 
4. B. Adamczewski, Y. Bugeaud, and F. Luca. Sur la complexité des nombres algébriques. C. R. Math. Acad. Sci. Paris, 339:11-14, 2004.

5. B. Adamczewski and N. Rampersad. On patterns occurring in binary algebraic numbers. Proc. Amer. Math. Soc., 136:3105-3109, 2008.

6. S. Akiyama, C. Frougny, and J. Sakarovitch. Powers of rationals modulo 1 and rational base number systems. Israel J. Math., 168:53-91, 2008.

7. B. Alexeev. Minimal DFA for testing divisibility. J. Comput. System Sci., 69(2):235-243, 2004.

8. J.-P. Allouche. Sur la complexité des suites infinies. Bull. Belg. Math. Soc., 1:133143, 1994.

9. J.-P. Allouche, E. Cateland, W. J. Gilbert, H.-O. Peitgen, J. Shallit, and G. Skordev. Automatic maps in exotic numeration systems. Theory Comput. Systems, 30:285-331, 1997.

10. J.-P. Allouche, J. Shallit, and G. Skordev. Self-generating sets, integers with missing blocks, and substitutions. Discrete Math., 292(1-3):1-15, 2005.

11. J.-P. Allouche and J. O. Shallit. The ubiquitous Prouhet-Thue-Morse sequence. In C. Ding, T. Helleseth, and H. Niederreiter, editors, Sequences and Their Applications, Proceedings of SETA '98, pages 1-16. Springer-Verlag, 1999.

12. J.-P. Allouche and J. O. Shallit. Automatic Sequences, Theory, Applications, Generalizations. Cambridge University Press, 2003.

13. G. Barat, V. Berthé, P. Liardet, and J. Thuswaldner. Dynamical directions in numeration. Ann. Inst. Fourier (Grenoble), 56:1987-2092, 2006.

14. M.-P. Béal, S. Lombardy, and J. Sakarovitch. Conjugacy and equivalence if weighted automata and functional transducers. In Proc. of CSR'06, volume 3967 of Lecture Notes in Computer Science, pages 58-69, 2006.

15. C. Berge. Graphes et hypergraphes. Dunod, Paris, 1973. Deuxième édition, Collection Dunod Université, Série Violette, No. 604.

16. E. R. Berlekamp, J. H. Conway, and R. K. Guy. Winning ways for your mathematical plays. Vol. 1. A K Peters Ltd., Natick, MA, second edition, 2001.

17. J. Berstel and J. Karhumäki. Combinatorics on words: a tutorial. Bull. European Assoc. Theor. Comput. Sci., 79:178-228, 2003.

18. V. Berthé. Autour du système de numération d'Ostrowski. Bull. Belg. Math. Soc. Simon Stevin, 8:209-239, 2001.

19. V. Berthé and M. Rigo, editors. Combinatorics, Automata and Number Theory, volume 135 of Encyclopedia of Mathematics and its Applications. Cambridge University Press, 2010.

20. V. Berthé and A. Siegel. Tilings associated with beta-numeration and substitutions. Integers, 5(3):A2, 46 pp. (electronic), 2005.

21. B. Boigelot, L. Bronne, and S. Rassart. An improved reachability analysis method for strongly linear hybrid systems. In Proc. 9th CAV, volume 1254 of Lecture Notes in Computer Science, pages 167-177, 1997.

22. B. Boigelot and J. Brusten. A generalization of Cobham's theorem to automata over real numbers. Theoret. Comput. Sci., 410(18):1694-1703, 2009.

23. B. Boigelot, J. Brusten, and J. Leroux. A generalization of Semenov's theorem to automata over real numbers. In R. A. Schmidt, editor, Automated Deduction, 22nd International Conference, CADE 2009, McGill University, Montreal, volume 5663 of Lecture Notes in Computer Science, pages 469-484, 2009.

24. B. Boigelot, S. Jodogne, and P. Wolper. An effective decision procedure for linear arithmetic over the integers and reals. ACM Trans. Comput. Log., 6(3):614-633, 2005. 
25. B. Boigelot, S. Rassart, and P. Wolper. On the expressiveness of real and integer arithmetic automata. In Proc. 25th ICALP (Aalborg), volume 1443 of Lecture Notes in Computer Science, pages 152-163. Springer, 1998.

26. D. W. Boyd. Irreducible polynomials with many roots of maximal modulus. Acta Arith., 68(1):85-88, 1994.

27. S. Brlek. Enumeration of factors in the Thue-Morse word. Discrete Appl. Math., 24:83-96, 1989.

28. V. Bruyère and G. Hansel. Bertrand numeration systems and recognizability. Theoret. Comput. Sci., 181:17-43, 1997.

29. V. Bruyère, G. Hansel, C. Michaux, and R. Villemaire. Logic and p-recognizable sets of integers. Bull. Belg. Math. Soc., 1:191-238, 1994.

30. Y. Bugeaud and J.-H. Evertse. On two notions of complexity of algebraic numbers. Acta Arith., 133:221-250, 2008.

31. E. Charlier, T. Kärki, and M. Rigo. Multidimensional generalized automatic sequences and shape-symmetric morphic words. Discrete Math., 310:1238-1252, 2010.

32. E. Charlier, N. Rampersad, M. Rigo, and L. Waxweiler. State complexity of testing divisibility. 2010. preprint.

33. E. Charlier, M. Rigo, and W. Steiner. Abstract numeration systems on bounded languages and multiplication by a constant. Integers, 8:A35, 19, 2008.

34. C. Choffrut and J. Karhumäki. Combinatorics of words. In G. Rozenberg and A. Salomaa, editors, Handbook of formal languages, volume vol. 1: word, language, grammar, pages 329-438. Springer-Verlag New York, Inc., 1997.

35. A. Cobham. On the Hartmanis-Stearns problem for a class of tag machines. In IEEE Conference Record of 1968 Ninth Annual Symposium on Switching and Automata Theory, pages 51-60, 1968.

36. A. Cobham. On the base-dependence of sets of numbers recognizable by finite automata. Math. Systems Theory, 3:186-192, 1969.

37. A. Cobham. Uniform tag sequences. Math. Systems Theory, 6:164-192, 1972.

38. D. Crisp, W. Moran, A. Pollington, and P. Shiue. Substitution invariant cutting sequences. J. Théor. Nombres Bordeaux, 5(1):123-137, 1993.

39. A. de Luca and S. Varricchio. On the factors of the Thue-Morse word on three symbols. Inform. Process. Lett., 27:281-285, 1988.

40. H. Derksen. A Skolem-Mahler-Lech theorem in positive characteristic and finite automata. Invent. Math., 168:175-224, 2007.

41. E. Duchêne, A. S. Fraenkel, R. Nowakowski, and M. Rigo. Extensions and restrictions of Wythoff's game preserving Wythoff's sequence as set of P-positions. J. Combinat. Theory Ser. A, 117:545-567, 2010.

42. E. Duchêne and M. Rigo. Invariant games. Theoret. Comput. Sci. to appear.

43. E. Duchêne and M. Rigo. Cubic Pisot unit combinatorial games. Monat. für Math., 155:217-249, 2008.

44. E. Duchêne and M. Rigo. A morphic approach to combinatorial games : the tribonacci case. Theor. Inform. Appl., 42:375-393, 2008.

45. F. Durand. A generalization of Cobham's theorem. Theory Comput. Syst., 31(2):169-185, 1998

46. F. Durand. Sur les ensembles d'entiers reconnaissables. J. Théor. Nombres Bordeaux, 10(1):65-84, 1998.

47. F. Durand. A theorem of Cobham for non primitive substitutions. Acta Arith., 104:225-241, 2002.

48. F. Durand and M. Rigo. On Cobham's theorem. In Handbook, Automata: from Mathematics to Applications. Europ. Math. Soc. publishing house. to appear. 
49. F. Durand and M. Rigo. Syndeticity and independent substitutions. Adv. in Appl. Math., 42:1-22, 2009.

50. S. Eilenberg. Automata, Languages, and Machines, volume A. Academic Press, 1974.

51. S. Ferenczi and C. Mauduit. Transcendence of numbers with a low complexity expansion. J. Number Theory, 67:146-161, 1997.

52. A. S. Fraenkel. Complementary iterated floor words and the flora game. SIAM J. on Discrete Math. to appear.

53. A. S. Fraenkel. How to beat your Wythoff games' opponent on three fronts. Amer. Math. Monthly, 89:353-361, 1982.

54. A. S. Fraenkel. Systems of numeration. Amer. Math. Monthly, 92:105-114, 1985.

55. A. S. Fraenkel. the Sprague-Grundy function for Wythoff's game. Theoret. Comput. Sci., 75:311-333, 1990.

56. A. S. Fraenkel. Heap games, numeration systems and sequences. Annals of Combin., 2:197-210, 1998.

57. A. S. Fraenkel. Complexity, appeal and challenges of combinatorial games. Theoret. Comput. Sci., 313(3):393-415, 2004.

58. C. Frougny. Representations of numbers and finite automata. Math. Systems Theory, 25:37-60, 1992.

59. C. Frougny. On the sequentiality of the successor function. Inform. and Comput., 139(1):17-38, 1997.

60. C. Frougny and B. Solomyak. Finite beta-expansions. Ergod. Th. ES Dynam. Sys., 12:713-723, 1992.

61. P. Grabner, C. Heuberger, and H. Prodinger. Distribution results for low-weight binary representations for pairs of integers. Theoret. Comput. Sci., 319:307-331, 2004.

62. G. Hansel and T. Safer. Vers un théorème de Cobham pour les entiers de Gauss. Bull. Belg. Math. Soc. Simon Stevin, 10:723-735, 2003.

63. G. H. Hardy and E. M. Wright. An Introduction to the Theory of Numbers. Oxford University Press, 5th edition, 1985.

64. J. Hartmanis and R. E. Stearns. On the computational complexity of algorithms. Trans. Amer. Math. Soc., 117:285-306, 1965.

65. M. Hollander. Greedy numeration systems and regularity. Theory Comput. Systems, 31:111-133, 1998.

66. J. Honkala. On the simplification of infinite morphic words. Theoret. Comput. Sci., 410(8-10):997-1000, 2009.

67. T. Kärki, A. Lacroix, and M. Rigo. On the recognizability of self-generating sets. J. Integer Sequences, 2010. Article 10.2.2.

68. I. Kátai and J. Szabó. Canonical number systems for complex integers. Acta Sci. Math. (Szeged), 37(3-4):255-260, 1975.

69. C. Kimberling. A self-generating set and the golden mean. J. Integer Seq., 3(2):Article 00.2.8, 2000 .

70. D. E. Knuth. The art of computer programming. Vol. 2. Addison-Wesley Publishing Co., Reading, Mass., second edition, 1981. Seminumerical algorithms, AddisonWesley Series in Computer Science and Information Processing.

71. P. B. A. Lecomte and M. Rigo. Numeration systems on a regular language. Theory Comput. Systems, 34:27-44, 2001.

72. H. Lenstra. Profinite Fibonacci numbers. Nieuw Arch. Wiskd. (5), 6(4):297-300, 2005. Also, in Europ. Math. Soc. Newsletter 61, Sept. 2006, 15-18.

73. D. Lind and B. Marcus. An Introduction to Symbolic Dynamics and Coding. Cambridge University Press, 1995. 
74. C. Löding. Efficient minimization of deterministic weak $\omega$-automata. Inform. Process. Lett., 79(3):105-109, 2001.

75. N. Loraud. $\beta$-shift, systèmes de numération et automates. J. Théorie Nombres Bordeaux, 7:473-498, 1995.

76. M. Lothaire. Algebraic Combinatorics on Words, volume 90 of Encyclopedia of Mathematics and Its Applications. Cambridge University Press, 2002.

77. M. Lothaire. Applied Combinatorics on Words, volume 105 of Encyclopedia of Mathematics and Its Applications. Cambridge University Press, 2005.

78. C. Michaux and R. Villemaire. Open questions around Büchi and Presburger arithmetics. In Logic: from foundations to applications (Staffordshire, 1993), Oxford Sci. Publ., pages 353-383. Oxford Univ. Press, New York, 1996.

79. J.-J. Pansiot. Complexité des facteurs des mots infinis engendrés par morphismes itérés. In Automata, languages and programming (Antwerp, 1984), volume 172 of Lecture Notes in Computer Science, pages 380-389. Springer, Berlin, 1984.

80. W. Parry. On the $\beta$-expansions of real numbers. Acta Math. Acad. Sci. Hung., 11:401-416, 1960.

81. D. Perrin. Finite automata. In J. van Leeuwen, editor, Handbook of Theoretical Computer Science, Volume B:Formal Models and Semantics, pages 1-57. Elsevier - MIT Press, 1990.

82. N. Pytheas Fogg. Substitutions in Dynamics, Arithmetics and Combinatorics, volume 1794 of Lecture Notes in Mathematics. Springer-Verlag, 2002. Ed. by V. Berthé and S. Ferenczi and C. Mauduit and A. Siegel.

83. M. Rigo and A. Maes. More on generalized automatic sequences. J. Automata, Languages, and Combinatorics, 7:351-376, 2002.

84. M. Rigo and L. Waxweiler. A note on syndeticity, recognizable sets and Cobham's theorem. Bull. European Assoc. Theor. Comput. Sci., 88:169-173, February 2006.

85. J. Sakarovitch. Éléments de théorie des automates. Vuibert, 2003. English corrected edition: Elements of Automata Theory, Cambridge University Press, 2009.

86. O. Salon. Suites automatiques à multi-indices. In Séminaire de Théorie des Nombres de Bordeaux, pages 4.01-4.27, 1986-1987.

87. O. Salon. Suites automatiques à multi-indices et algébricité. C. R. Acad. Sci. Paris, 305:501-504, 1987.

88. W. M. Schmidt. Diophantine Approximation, volume 785 of Lecture Notes in Mathematics. Springer-Verlag, 1980.

89. J. O. Shallit. Numeration systems, linear recurrences, and regular sets. Inform. Comput., 113:331-347, 1994.

90. C. E. Silva. Invitation to ergodic theory, volume 42 of Student Mathematical Library. American Mathematical Society, Providence, RI, 2008.

91. M. Waldschmidt. Diophantine approximation on linear algebraic groups, volume 326 of Grundlehren der Mathematischen Wissenschaften. Springer-Verlag, Berlin, 2000. Transcendence properties of the exponential function in several variables.

92. M. Waldschmidt. Words and transcendence. In Analytic Number Theory - Essays in Honour of Klaus Roth, chapter 31, pages 449-470. Cambridge University Press, 2009. http://arxiv.org/abs/0908.4034 